\title{
AXL RTK-a therapeutic target in RCC?
}

New data, published by Yu and colleagues in the British Journal of Cancer, have shown that AXL oncogene protein (AXL) is upregulated in renal cell carcinoma (RCC) and that targeting it using a humanized AXL antibody_hMAb173 - significantly reduces tumour growth, decreases cell proliferation and induces apoptosis in vivo. AXL belongs to the TYRO3, AXL and MERTK family of receptor tyrosine kinases (RTKs) and is overexpressed in RCC. Signalling through AXL promotes cancer cell survival, proliferation, migration and invasion.

Yu et al. analysed AXL expression in 174 RCC samples using immunohistochemistry; 54 of 110 clear cell RCC samples and 49 of 64 papillary RCC samples showed moderate or strong staining and, overall, $20 \%$ of RCC samples had moderate or high staining for AXL; all four samples from normal kidney showed no staining. In 11 paired samples of RCC and normal kidney tissue, eight RCC samples showed high AXL expression on Western blot compared with low or no expression in the corresponding normal tissue samples.

Investigations into AXL expression in the 786-O, 769-P and ACHN kidney cancer cell lines by quantitative, real-time PCR revealed that expression was relatively high in all three compared with expression in the embryonic kidney cell line, 293T. Knockdown of AXL in the 786-O and ACHN cell lines using short interfering RNA effectively decreased AXL expression and reduced cell attachment, viability and invasion in culture. Signalling-pathway analysis showed that phosphorylation of ribosomal protein S6 kinase and RAC-gamma serine/threonine-protein kinase, which are key signalling molecules in the phosphatidylinositol 4,5-bisphosphate 3-kinase pathway, was also substantially reduced.

The group then humanized MAb173, a monoclonal antibody against AXL that they had previously developed, to test the ability of this antibody to target kidney cancer. In vitro analysis showed that hMAb173 degraded AXL, with expression reducing by $80 \%$ after $3 \mathrm{~h}$ of incubation at $37^{\circ} \mathrm{C}$. In an ex vivo tumour model, incubation with hMAb173 for 2 days induced apoptosis. In vivo treatment of 786-O xenograft tumour models with hMAb173 inhibited tumour growth by $78 \%$ compared with controls $(P<0.002)$. AXL was barely detectable in the hMAb173-treated tumours and the anti-AXL antibody promoted tumour cell apoptosis and reduced cell proliferation and ribosomal protein S6 kinase phosphorylation.

Drs Liu and Gill, corresponding authors for the study, told Nature Reviews Urology:

"Targeting AXL with a highly specific monoclonal antibody is advantageous as signalling through other AXL family members, which are expressed in normal organs, will not be affected." They continued "We are working to enhance the efficacy of this AXL antibody," concluding "in the future, staining tumours for AXL will enable selection of patients suitable for this therapy."

Louise Stone

Original article $\mathrm{Yu}, \mathrm{H}$. et al. Axl receptor tyrosine kinase is a potential therapeutic target in renal cell carcinoma. Br. J. Cancer doi:10.1038/bjc.2015.237 